\title{
The phylogeny of the Anderson's White-bellied Rat (Niviventer andersoni) based on complete mitochondrial genomes provides insight into its evolutionary history
}

\author{
Shujing Liu ${ }^{1}$, Lili Fu ${ }^{2}$, Jihua Zhou ${ }^{3}$, Jizhou Lv${ }^{4}$, Zhongyang Tan ${ }^{1}$, Yunzhi Zhang ${ }^{2}$, and \\ Xingyi $\mathrm{Ge}^{1}$ \\ ${ }^{1}$ Hunan University \\ ${ }^{2}$ Dali University \\ ${ }^{3}$ Yunnan Institute of Endemic Diseases Control and Prevention \\ ${ }^{4}$ Chinese Academy of Inspection and Quarantine Institute of Animal Quarantine
}

July 7,2021

\begin{abstract}
Anderson's White-bellied Rat, Niviventer andersoni (Thomas, 1911) (Muridae, Niviventer) is an species endemic to China. In the present study, we have sequenced the first complete mitochondrial genome of $N$. andersoni using next-generation sequencing. The 16,291 bp mitochondrial genome consists of 22 transfer RNA genes, 13 protein-coding genes (PCGs), two ribosomal RNA genes, and one non-coding control region (D-Loop). Phylogenetic analyses of the nucleotide sequences of all 13 PCGs, PCGs minus ND6 and the entire mitogenome sequence except for the D-loop, produce nearly identical, well-resolved topologies. Our results support that $N$. andersoni clustered with $N$. excelsior and form a sister group with $N$. confucianus, and they statistically reject the hypothesis from one cytochrome b (cytb) gene tree that $N$. confucianus is sister to $N$. fulvescens. Our research may be helpful to further reconsideration of clearer taxonomy and improve our understanding of mitogenomic evolution in the genus Niviventer.
\end{abstract}

\section{Introduction}

Anderson's White-bellied Rat, Niviventer andersoni belongs to genus Niviventer, family Muridae, and order Rodentia. N. andersoni is a species endemic to China, and has the largest body-size when compared with congeneric species of Niviventer [1]. They live in various kinds of forest in both lowlands and mountains [2]. Fossil records showed that this species extended to the low altitude regions of Southeast China during the late Quaternary in Chongqing and Guizhou Provinces, suggesting its potential to expand southward the climate turned colder $[3,4]$.

Niviventer contains 17 recognized species, with another 65 are recognized as synonyms, occurring from the Himalayas and China to the Great Sunda Islands [5]. All Niviventer species are distinguished from other murid rodents by the long, slender, flat craniums and the tail-tip on tails [6]. They inhabit a variety of habitats ranging from damp forests to dry valleys. They are also among the most common infectious agents in humans [7]. According to previous studies (Musser, 1981), the Niviventer was separated into two primary divisions: the $N$. andersoni -Division and the $N$. niviventer -Division [8]. Phylogenetic trees based on mitochondrial cytochrome b (cytb) gene showed that $N$. andersoniand $N$. excelsior were clustered together and comprised the $N$. andersoni-Division [6]. Meanwhile, N. fulvescensand N. cremoriventer were initially clustered together, with $N$. confucianus as the next closest relative and formed the sister group of, $N$. niviventer -Division. However, single gene sequences are sometimes limited in their provision of useful 
data, since each gene evolves under different evolutionary pressures and time scales [9]. Compared to single mitochondrial gene sequences, complete mitochondrial genome sequences can provide improved resolution and sensitivity for investigations into the evolutionary relationships between closely related species [21,24].

Up to now, the complete mitogenomes of 4 species within the genusNiviventer were available in GenBank. Complete mitochondrial genomes have been used for taxonomic and phylogenetic analyses of diverse animal groups, due to its small size, maternal inheritance, low level of recombination and fast rate of evolution (particularly in rodents) [10-13]. The lack of genetic data has limited our understanding of the phylogeny of $N$. andersoni. In the present study, we sequenced the complete mitochondrial genome of $N$. andersoni

- The study has provided the features of the $N$. andersoni mitochondrial genome and has allowed us to compare its phylogenetic relationships with several other rat species. Our findings provide useful genetic data for phylogenetic comparisons to other rodent species with complete mitogenome information.

\section{Materials and methods}

\subsection{Sample collection and genomic DNA extraction}

Individuals of $N$. andersoni were collected from Lufeng County, Yunnan province, China $\left(24^{\circ} 57^{\prime} 45.774^{\prime \prime}\right.$; 102deg10'15.7296", $\mathrm{H}=1875.43 \mathrm{~m}$ ), in August 2018. These individuals were sacrificed and dissected for organ collection. The heart, liver, spleen, lung, kidney and muscle were kept in the cryopreservation tubes directly. All the samples were immediately put in liquid nitrogen for short storage, then transported to the laboratory in dry ice and stored at -80degC. DNA was extracted from the muscle using mitochondrial extraction kit (Solarbio) and stored at $-80 \operatorname{deg}$.

2.2 Mitogenome sequencing, assembly and annotation

The mitochondrial DNA was subjected to random PCR (rPCR) as previously described [14]. The purified rPCR products were used to construct the sequencing library and sequenced on HiSeq-PE150 instrument (TIANGEN, Beijing, China). The raw reads were trimmed and filtered using Trimmomatic (Version 0.39) [15]. The cleaned reads were aligned to NCBI non-redundant protein sequence database using BLASTx by DIAMOND [16]. Mitochondrial reads were picked and de novo assembled into a complete mitochondrial genome using Geneious software package (Version 2019.1.1) [17]. Protein coding genes (PCGs) were annotated using the NCBI ORF Finder (https://www.ncbi.nlm.nih.gov/orffinder/) and BLASTx with the vertebrate mitochondrial genetic code. The tRNA genes were identified using the tRNAscan-SE Search Server under the default search mode, using the vertebrate mitochondrial genetic code source [18]. Composition skew analysis was calculated according to the formulas: AT skew $=(\mathrm{A}-\mathrm{T}) /(\mathrm{A}+\mathrm{T})$ and $\mathrm{GC}$ skew $=(\mathrm{G}-\mathrm{C}) /(\mathrm{G}+\mathrm{C})[19]$. Relative synonymous codon usage (RSCU) values were calculated using CodonW 1.4.2 [20]. The circular mitochondrial genome map of $N$. andersoni was drawn using OGDRAW 1.3.1 [21].

\subsection{Phylogenetic analysis}

Phylogenetic analysis was performed comparing $N$. andersoni and 12 other rat mitogenomes downloaded from GenBank (Table 1). The nucleotide sequences were aligned using ClustalX with default settings before concatenation by DAMBE (Version 7.2) [22,23]. Models of evolution were evaluated using corrected Aikake Information Criteria (AICc) in jModelTest 2.1.10 to determine the best nucleotide substitution model [24]. Maximum likelihood (ML) analysis of the 13 PCGs in 13 species of rat was also performed using MEGA X [25]. The support values of the ML tree were evaluated via a bootstrap test with 1,000 iterations.

\section{Results and discussion}

\subsection{Genome organization}

After quality filtering the raw reads, a total of 1,578,672 high quality clean reads were obtained and used to assemble the $N$. andersonimitochondrial genome. After obtaining the complete mitochondrial genome sequence of $N$. andersoni we deposited it in NCBI with GenBank accession number MW030174. The mitogenome of $N$. andersoni was a circular DNA molecule which was 16,291 bp in length. As shown in Fig. 1, the mitogenome organization of $N$. andersoni was similar to that of most all other rodents [26]. 
Thirty-seven typical mitochondrial genes were identified, including 13 PCGs, 22 tRNAs and 2 rRNAs (Table 2). Most of genes were encoded on the Heavy $(\mathrm{H})$-strand, while ND6 and 8 tRNAs were encoded on the Light (L)-strand.

The total base composition of $N$. andersoni mitochondrial genome was estimated to be $33.7 \%$ for A, $25.8 \%$ for C, $12.1 \%$ for $\mathrm{G}$ and $30.0 \%$ for $\mathrm{T}$, which makes as AT and GC percentage of $61.6 \%$ and $38.4 \%$, respectively, indicating that the mitochondrial genome is biased towards AT (Table 3). Such base composition biases have been reported to play a vital role in the replication and transcription of mitochondrial genome [27]. It also showed a negative GC skew value (-0.347), indicating that $\mathrm{C}$ is more common than $\mathrm{G}$ whereas the AT skewness was positive (0.092) suggesting that A occurs more frequently than $\mathrm{T}$ in the $N$. andersoni mitochondrial genome (Table 3).

\subsection{Protein-coding genes (PCGs)}

Total length of the 13 PCGs was 11,420 bp, which accounted for $70.1 \%$ of the mitogenome. Initiation codons of all PCGs in mitogenome of $N$. andersoni were typical ATN, except for ND1, which started with GTG. All PCGs of the mitogenome of $N$. andersoni terminated with complete (TAA) or truncated (T) stop codons, except for ND2 which terminated with CAT (Table 2). The relative synonymous codon usage (RSCU) values of PCGs are displayed in Table 4, which also shows that the protein-coding gene region has 3,805 codons. According to the RSCU analyses, CUA (L), AUU (I) and AUA (M) were the three most frequently used codons. Leucine, isoleucine and threonine were the most frequent PCG amino acids (Fig. 2). This may explain the negative GC-skew and positive AT-skew of PCGs.

\subsection{Ribosomal RNA and Transfer RNA genes}

The mitogenome of $N$. andersoni contained the typical 22 tRNA genes throughout the genome and appeared to be highly $\mathrm{A}+\mathrm{T}$ biased, ranging in length from $59 \mathrm{bp}$ to $75 \mathrm{bp}$. Among these tRNA genes, eight tRNAs were encoded on the L-strand and the remaining 14 were encoded on the H-strand (Table 2). All the tRNA genes exhibited a typical cloverleaf structure, except trns1, which lacked a dihydroxyuridine arm that had been simplified to a ring shape. Loss of the DHU arm is common in the mitogenomes of many mammal animals [28].

The two rRNA genes (lrRNA, srRNA) encoding the small and large ribosomal subunits, were identified on the L- strand of $N$. andersoni, and were located between tRNA $^{\text {Phe }}$ and tRNA ${ }^{\text {Leu }}$. The $\operatorname{lrRNA}$ and srRNA lengths are 1,567 and $957 \mathrm{bp}$, respectively. The A+T content of rRNA was 63.43\%, and its AT-skew (0.204) and GC-skew (-0.099) showed that more As and Cs were present in the rRNA than As and Gs (Table 3).

\subsection{Phylogenetic analysis}

Based on 13 PCGs of 13 rat species, we established a phylogenetic tree by maximum likelihood method with 1,000 replications which set Mus musculus as outgroup (Fig. 3A). Some researchers have suggested that ND6 gene sequences should be excluded during phylogenetic analysis due to its high heterogeneity and consistently poor phylogenetic performance [29]. Thus, we constructed another phylogenetic tree based on PCGs excluding ND6 (Fig. 3B). The results of the two phylogenetic analyses were almost the same. When compared with other rat species, $N$. andersoni was phylogenetically closer to $N$. excelsior and clustered within genus Niviventer .

To further investigate the phylogenetic relationships of $N$. andersoni, the phylogenetic relationships were reconstructed based on the complete mitochondrial genome (Fig. 4). 13 species were used to preform phylogenetic analysis (Table 1). The D-loop region was excluded because of the rapid mutation rate in this region. The maximum likelihood tree was constructed based on the complete mitochondrial genome (except D-loop). The topologies of the maximum likelihood trees constructed based on the complete sequence and PCGs of the mitochondrial genome were identical.Our results were generally congruent with those from the previous study using only the cytb gene, except for the phylogenetic position of $N$. confucianus - Single cytb gene trees in previous studies showed that $N$. confucianus was closer to $N$. fulvescens and $N$. cremoriventer than to $N$. andersoni and $N$. excelsior $[6,30,31]$. Our results suggest that $N$. andersoni and 
$N$. excelsior clustered together, then with $N$. confucianus, and formed a sister group of $N$. fulvescens and $N$. cremoriventer. Since each gene evolves under different evolutionary pressure and time scale, it has been known that one gene tree for a population may differ from other gene trees for the same population depending on the subjective selection of the genes [9]. The single mitochondrial gene tree and complete mitogenome tree were conflicting, suggesting that phylogenetic tree using complete mitochondrial genomes was warranted.

\section{Conclusion}

We have sequenced the complete mitochondrial genome of $N$. andersoni for the first time and compared it with closely related species of the family Muridae. The mitochondrial genome structural features were similar to other species in genus Niviventer. In the phylogenetic analysis of sequences of the 13 PCGs, the PCGs excluding ND6 and the complete mitogenome without D-loop, $N$. andersoni was consistently the most similar to $N$. excelsior, consistent with previous studies based on single cytb gene sequences and morphological characteristics. Phylogenetic analysis based on the complete mitogenome showed that $N$. confucianus had the closest relationship to $N$. andersoni and $N$. excelsior, rather than $N$. fulvescens and $N$. cremoriventer as previously suggested. The availability of complete mitochondrial genome of $N$. andersoni should be helpful to better understand evolution within the genus Niviventer, as well as its relationship to other murid rodents.

\section{Acknowledgements and declaration of interest}

This work was jointly funded by the National Key Research and Development Program of China "Research and application of national quality infrastructure" (project/subject SN. 2017YFF0210302), and the National Natural Science Foundation of China (81660558) and the Yunnan health training project of high-level talents (L-2017027). The authors declare that there is no conflict of interests.

\section{Ethics approval and consent to participate}

Sample collection and all the experiments in this study were under the ethics approval by the Yunnan Institute of Endemic Disease Control and Prevention with the animal ethics approval number: DLDXLL2017007.

\section{Data accessibility}

The following information was supplied regarding the availability of DNA sequences: The complete mitogenome of Niviventer andersoni is deposited in GenBank of NCBI under accession number MW030174.

\section{References}

1. Ge D, Lu L, Cheng J, Xia L, Chang Y, Wen Z, Lv X, Du Y, Liu Q, Yang Q (2017) An endemic rat species complex is evidence of moderate environmental changes in the terrestrial biodiversity centre of China through the late Quaternary. Scientific reports 7:46127. doi:10.1038/srep46127

2. Li S, Yang J (2009) Geographic variation of the Anderson's Niviventer (Niviventer andersoni) (Thomas, 1911) (Rodentia: Muridae) of two new subspecies in China verified with cranial morphometric variables and pelage characteristic. Zootaxa 2196:48-58

3. Bekken D, Schepartz LA, Miller-Antonio S, Yamei H, Weiwen H (2004) Taxonomic abundance at Panxian Dadong, a middle Pleistocene Cave in South China. Asian Perspect 43:333-359

4. Bahain JJ (2007) Current research in Chinese pleistocene archaeology. Anthropologie 111 (1):134-135

5. Wilson DE, Reeder DM (2005) Mammal Species of the World. A Taxonomic and Geographic Reference (3rd ed). Johns Hopkins University Press, 2142 pp,

6. Jing M, Yu HT, Wu SH, Wang W, Zheng X (2007) Phylogenetic relationships in genus Niviventer (Rodentia: Muridae) in China inferred from complete mitochondrial cytochrome b gene. Molecular phylogenetics and evolution 44 (2):521-529. doi:10.1016/j.ympev.2007.04.003 
7. Keesing F, Belden LK, Daszak P, Dobson A, Harvell CD, Holt RD, Hudson P, Jolles A, Jones KE, Mitchell CE, Myers SS, Bogich T, Ostfeld RS (2010) Impacts of biodiversity on the emergence and transmission of infectious diseases. Nature 468 (7324):647-652. doi:10.1038/nature09575

8. Musser GG (1981) Results of the Archbold Expeditions. No. 105. Notes on systematics of Indo-Malayan murid rodents, and descriptions of new genera and species fromCeylon, Sulawesi, and the Philippines. Bulletin of the American Museum of Natural History 168((3):236-256

9. Choi J, Kim SH (2017) A genome Tree of Life for the Fungi kingdom. Proceedings of the National Academy of Sciences of the United States of America 114 (35):9391-9396. doi:10.1073/pnas.1711939114

10. Kim T, Lee Y, Kil HJ, Park JK (2020) The mitochondrial genome of Acrobeloides varius (Cephalobomorpha) confirms non-monophyly of Tylenchina (Nematoda). PeerJ 8:e9108. doi:10.7717/peerj.9108

11. Wei HX, Li FJ, Wang XM, Wang Q, Chen GY, Zong H, Chen SD (2017) The characterization of complete mitochondrial genome and phylogenetic relationship within Rattus genus (Rodentia: Muridae). Biochem Syst Ecol 71:179-186. doi:10.1016/j.bse.2017.01.012

12. Ladoukakis ED, Zouros E (2017) Evolution and inheritance of animal mitochondrial DNA: rules and exceptions. Journal of biological research 24:2. doi:10.1186/s40709-017-0060-4

13. Lavrov DV, Pett W (2016) Animal Mitochondrial DNA as We Do Not Know It: mt-Genome Organization and Evolution in Nonbilaterian Lineages. Genome biology and evolution 8 (9):2896-2913. doi:10.1093/gbe/evw195

14. Li Y, Ge X, Zhang H, Zhou P, Zhu Y, Zhang Y, Yuan J, Wang LF, Shi Z (2010) Host range, prevalence, and genetic diversity of adenoviruses in bats. Journal of virology 84 (8):3889-3897. doi:10.1128/JVI.02497-09

15. Bolger AM, Lohse M, Usadel B (2014) Trimmomatic: a flexible trimmer for Illumina sequence data. Bioinformatics 30 (15):2114-2120. doi:10.1093/bioinformatics/btu170

16. Buchfink B, Xie C, Huson DH (2015) Fast and sensitive protein alignment using DIAMOND. Nature methods 12 (1):59-60. doi:10.1038/nmeth.3176

17. Kearse M, Moir R, Wilson A, Stones-Havas S, Cheung M, Sturrock S, Buxton S, Cooper A, Markowitz S, Duran C, Thierer T, Ashton B, Meintjes P, Drummond A (2012) Geneious Basic: an integrated and extendable desktop software platform for the organization and analysis of sequence data. Bioinformatics 28 (12):1647-1649. doi:10.1093/bioinformatics/bts199

18. Chan PP, Lowe TM (2019) tRNAscan-SE: Searching for tRNA Genes in Genomic Sequences. Methods in molecular biology 1962:1-14. doi:10.1007/978-1-4939-9173-0_1

19. Perna NT, Kocher TD (1995) Patterns of nucleotide composition at four fold degenerate sites of animal mitochondrial genomes. Journal of Molecular Evolution 41:353-358

20. Wu XM, Wu SF, Ren DM, Zhu YP, He FC (2007) [The analysis method and progress in the study of codon bias]. Yi chuan $=$ Hereditas 29 (4):420-426. doi:10.1360/yc-007-0420

21. Greiner S, Lehwark P, Bock R (2019) OrganellarGenomeDRAW (OGDRAW) version 1.3.1: expanded toolkit for the graphical visualization of organellar genomes. Nucleic acids research 47 (W1):W59-W64. doi:10.1093/nar/gkz238

22. Xia X (2017) DAMBE6: New Tools for Microbial Genomics, Phylogenetics, and Molecular Evolution. The Journal of heredity 108 (4):431-437. doi:10.1093/jhered/esx033

23. Larkin MA, Blackshields G, Brown NP, Chenna R, McGettigan PA, McWilliam H, Valentin F, Wallace IM, Wilm A, Lopez R, Thompson JD, Gibson TJ, Higgins DG (2007) Clustal W and Clustal X version 2.0. Bioinformatics 23 (21):2947-2948. doi:10.1093/bioinformatics/btm404 
24. Darriba D, Taboada GL, Doallo R, Posada D (2012) jModelTest 2: more models, new heuristics and parallel computing. Nature methods 9 (8):772. doi:10.1038/nmeth.2109

25. Kumar S, Stecher G, Li M, Knyaz C, Tamura K (2018) MEGA X: Molecular Evolutionary Genetics Analysis across Computing Platforms. Molecular biology and evolution 35 (6):1547-1549. doi:10.1093/molbev/msy096

26. Boore JL (1999) Animal mitochondrial genomes. Nucleic acids research 27 (8):1767-1780. doi:10.1093/nar/27.8.1767

27. Wei SJ, Shi M, Chen XX, Sharkey MJ, van Achterberg C, Ye GY, He JH (2010) New views on strand asymmetry in insect mitochondrial genomes. PloS one 5 (9):e12708. doi:10.1371/journal.pone.0012708

28. Wolstenholme DR (1992) Animal mitochondrial DNA: structure and evolution. International review of cytology 141:173-216. doi:10.1016/s0074-7696(08)62066-5

29. Miya M, Nishida M (2000) Use of mitogenomic information in teleostean molecular phylogenetics: a tree-based exploration under the maximum-parsimony optimality criterion. Molecular phylogenetics and evolution 17 (3):437-455. doi:10.1006/mpev.2000.0839

30. Li Y, Li Y, Li H, Wang J, Rong X, Li Y (2020) Niviventer confucianus sacer (Rodentia, Muridae) is a distinct species based on molecular, karyotyping, and morphological evidence. ZooKeys 959:137-159. doi: $10.3897 /$ zookeys.959.53426

31. Ge D, Lu Li, Xia L, Du Y, Z hiXin Wen , Cheng J, Abramov AV, Yang Q (2018) Molecular phylogeny, morphological diversity, and systematic revision of a species complex of common wild rat species in China (Rodentia, Murinae). Journal of Mammalogy 99 (6):1350-1374. doi:10.1093/jmammal/gyy117

\section{Figure Legends}

Fig. 1. Mitochondrial genome map of Niviventer andersoni .Fig. 2. The relative synonymous codon usage (RSCU) of in the mitogenome of Niviventer andersoni. The box below the bar chart represents all codons encoding each amino acid, and the height of the column above represents the sum of all RSCU values.Fig. 3. The maximum likelihood analyses of phylogenetic relationship based on (A) 13 PCGs and (B) 12 PCGs of 13 rat species.

Fig. 4. The maximum likelihood analyses of phylogenetic relationships based on complete mitochondrial genome minus the D-loop.

Table 1 Complete mitochondrial genomes used for phylogenetic analysis in this study

Table 2 Characteristics of the mitochondrial genome of

Niviventer andersoni

Table 3 Nucleotide composition and AT-GC skewness of theNiviventer andersoni mitogenome

Table 4 Relative synonymous codon usage and codon numbers inNiviventer andersoni mitochondrial protein-coding genes

Table 1 Complete mitochondrial genomes used for phylogenetic analysis in this study

\begin{tabular}{llll}
\hline Genus & Species & Common name & GenBank \\
\hline Leopoldamys & Leopoldamys edwardsi & Edwards's long-tailed giant rat & NC_025670.1 \\
& Leopoldamys sabanus & long-tailed giant rat & MN964122.1 \\
Mus & Mus musculus & house mouse & NC_005089.1 \\
Niviventer & Niviventer confucianus & Chinese white-bellied rat & NC_023960.1 \\
& Niviventer cremoriventer & dark-tailed tree rat & NC_035822.1 \\
& Niviventer excelsior & large white-bellied rat & NC_019617.1
\end{tabular}




\begin{tabular}{llll}
\hline Genus & Species & Common name & GenBank \\
\hline \multirow{3}{*}{ Rattus } & Niviventer fulvescens & Chestnut white-bellied rat & NC_028715.1 \\
& Rattus andamanensis & Indochinese forest rat & NC_046686.1 \\
& Rattus baluensis & summit rat & NC_035621.1 \\
Rattus norvegicus & Norway rat & NC_001665.2 \\
& Rattus tanezumi & Oriental house rat & NC_011638.1 \\
& Rattus tiomanicus & Malayan field rat & MN126562.1 \\
\hline
\end{tabular}

\begin{tabular}{llllllll}
\hline Gene & Start & Stop & Length(bp) & Start Codon & Stop Codon & Strand & A+T \\
\hline tRNA-Phe & 1 & 68 & 68 & & & $\mathrm{H}$ & $67.6 \%$ \\
s-rRNA & 69 & 1025 & 957 & & & $\mathrm{H}$ & $62.6 \%$ \\
tRNA-Val & 1026 & 1093 & 68 & & & $\mathrm{H}$ & $58.8 \%$ \\
l-rRNA & 1094 & 2660 & 1567 & & & $\mathrm{H}$ & $63.9 \%$ \\
tRNA-Leu2 & 2661 & 2735 & 75 & & & $\mathrm{H}$ & $54.7 \%$ \\
ND1 & 2736 & 3690 & 955 & GTG & T- & $\mathrm{H}$ & $57.6 \%$ \\
tRNA-lle & 3691 & 3759 & 69 & & & $\mathrm{H}$ & $72.5 \%$ \\
tRNA-Gln & 3757 & 3827 & 71 & & & $\mathrm{~L}$ & $62.0 \%$ \\
tRNA-Met & 3831 & 3899 & 69 & & & $\mathrm{H}$ & $52.2 \%$ \\
ND2 & 3900 & 4935 & 1036 & ATC & CAT & $\mathrm{H}$ & $63.7 \%$ \\
tRNA-Trp & 4936 & 5001 & 66 & & & $\mathrm{H}$ & $63.6 \%$ \\
tRNA-Ala & 5003 & 5071 & 69 & & & $\mathrm{~L}$ & $69.6 \%$ \\
tRNA-Asn & 5073 & 5143 & 71 & & & $\mathrm{~L}$ & $66.2 \%$ \\
tRNA-Cys & 5178 & 5245 & 68 & & & $\mathrm{~L}$ & $50.0 \%$ \\
tRNA-Tyr & 5246 & 5311 & 66 & & $\mathrm{~L}$ & $54.5 \%$ \\
COX1 & 5313 & 6857 & 1545 & ATG & TAA & $\mathrm{H}$ & $59.7 \%$ \\
tRNA-Ser2 & 6855 & 6923 & 69 & & & $\mathrm{~L}$ & $59.4 \%$ \\
tRNA-Asp & 6927 & 6994 & 68 & & & $\mathrm{H}$ & $82.4 \%$ \\
COX2 & 6996 & 7679 & 684 & ATG & TAA & $\mathrm{H}$ & $59.5 \%$ \\
tRNA-Lys & 7683 & 7747 & 65 & & & $\mathrm{H}$ & $66.2 \%$ \\
ATP8 & 7748 & 7951 & 204 & ATG & TAA & $\mathrm{H}$ & $64.2 \%$ \\
ATP6 & 7909 & 8589 & 681 & ATG & TAA & $\mathrm{H}$ & $62.7 \%$ \\
COX3 & 8589 & 9372 & 784 & ATG & T- & $\mathrm{H}$ & $57.4 \%$ \\
tRNA-Gly & 9373 & 9440 & 68 & & & $\mathrm{H}$ & $64.7 \%$ \\
ND3 & 9441 & 9788 & 348 & ATC & TAA & $\mathrm{H}$ & $62.9 \%$ \\
tRNA-Arg & 9790 & 9857 & 68 & & & $\mathrm{H}$ & $80.9 \%$ \\
ND4L & 9860 & 10156 & 297 & ATG & TAA & $\mathrm{H}$ & $63.3 \%$ \\
ND4 & 10150 & 11527 & 1378 & ATG & T- & $\mathrm{H}$ & $62.0 \%$ \\
tRNA-His & 11528 & 11595 & 68 & & & $\mathrm{H}$ & $73.5 \%$ \\
tRNA-Ser1 & 11596 & 11654 & 59 & & $\mathrm{H}$ & $62.7 \%$ \\
tRNA-Leu1 & 11654 & 11724 & 71 & & & $\mathrm{H}$ & $66.2 \%$ \\
ND5 & 11725 & 13554 & 1830 & ATA & TAA & $\mathrm{H}$ & $61.3 \%$ \\
ND6 & 13532 & 14050 & 519 & ATG & TAA & $\mathrm{L}$ & $61.3 \%$ \\
tRNA-Glu & 14051 & 14119 & 69 & & $\mathrm{~L}$ & $69.6 \%$ \\
CYTB & 14125 & 15283 & 1159 & ATG & TAA & $\mathrm{H}$ & $58.3 \%$ \\
tRNA-Thr & 15269 & 15335 & 67 & & $\mathrm{H}$ & $68.7 \%$ \\
tRNA-Pro & 15336 & 15402 & 67 & & $\mathrm{H}$ & $62.7 \%$ \\
D-loop & 15403 & 16291 & 889 & & & $64.2 \%$ \\
\hline & & & & & &
\end{tabular}


Table 2 Characteristics of the mitochondrial genome of Niviventer andersoni

Table 3 Nucleotide composition and AT-GC skewness of the Niviventer andersoni mitogenome

\begin{tabular}{lllllllll}
\hline Niviventer andersoni & Size (bp) & $\mathrm{A}$ & $\mathrm{G}$ & $\mathrm{T}$ & $\mathrm{C}$ & $\mathrm{A}+\mathrm{T}$ & AT skewness & GC skewness \\
\hline mitogenome & 16291 & 33.65 & 12.53 & 27.97 & 25.85 & 61.62 & 0.092 & -0.347 \\
PCGs & 12309 & 28.96 & 11.51 & 27.29 & 25.01 & 56.25 & 0.030 & -0.370 \\
tRNAs & 1499 & 34.62 & 18.55 & 30.29 & 16.54 & 64.91 & 0.067 & 0.057 \\
rRNAs & 2524 & 38.19 & 16.48 & 25.24 & 20.09 & 63.43 & 0.204 & -0.099 \\
Control region & 889 & 34.31 & 11.36 & 29.92 & 24.41 & 64.23 & 0.068 & -0.365 \\
\hline
\end{tabular}

Table 4 Relative synonymous codon usage and codon numbers inNiviventer andersoni mitochondrial proteincoding genes

\begin{tabular}{llllllllllll}
\hline Codon & Count & RSCU & Codon & Count & RSCU & Codon & Count & RSCU & Codon & Count & RSCU \\
\hline UCA(S) & 115 & 1.83 & AUG(M) & 32 & 0.28 & GGU(G) & 35 & 0.67 & UAG(*) & 0 & 0.00 \\
UCC(S) & 72 & 1.14 & AUA(M) & 198 & 1.72 & GGG(G) & 22 & 0.42 & UAA $(*)$ & 8 & 4.00 \\
UCG(S) & 10 & 0.16 & AAC(N) & 102 & 1.31 & GGC(G) & 59 & 1.12 & AGA $(*)$ & 0 & 0.00 \\
UCU(S) & 55 & 0.87 & AAU(N) & 54 & 0.69 & GGA(G) & 94 & 1.79 & AGG $\left.*^{*}\right)$ & 0 & 0.00 \\
ACA(T) & 159 & 2.05 & CCU(P) & 35 & 0.70 & CAC(H) & 71 & 1.46 & GCU(A) & 67 & 1.11 \\
ACU(T) & 59 & 0.76 & CCG(P) & 4 & 0.08 & CAU(H) & 26 & 0.54 & GCG(A) & 8 & 0.13 \\
ACC(T) & 86 & 1.11 & CCC(P) & 52 & 1.04 & AUU(I) & 200 & 1.08 & GCC(A) & 91 & 1.50 \\
ACG(T) & 6 & 0.08 & CCA(P) & 109 & 2.18 & AUC(I) & 170 & 0.92 & GCA(A) & 76 & 1.26 \\
GUU(V) & 35 & 0.86 & CAA(Q) & 81 & 1.88 & AAA(K) & 94 & 1.86 & UGU(C) & 5 & 0.33 \\
GUG(V) & 15 & 0.37 & CAG(Q) & 5 & 0.12 & AAG(K) & 7 & 0.14 & UGC(C) & 25 & 1.67 \\
GUC(V) & 41 & 1.01 & CGA(R) & 43 & 2.69 & CUA(L) & 251 & 2.19 & GAU(D) & 25 & 0.66 \\
GUA(V) & 71 & 1.75 & CGC(R) & 14 & 0.88 & CUC(L) & 97 & 0.85 & GAC(D) & 51 & 1.34 \\
UGA(W) & 98 & 1.85 & CGG(R) & 1 & 0.06 & CUG(L) & 19 & 0.17 & GAG(E) & 13 & 0.28 \\
UGG(W) & 8 & 0.15 & CGU(R) & 6 & 0.38 & CUU(L) & 91 & 0.80 & GAA(E) & 81 & 1.72 \\
UAC(Y) & 69 & 1.08 & AGC(S) & 38 & 1.33 & UUA(L) & 116 & 1.76 & UUU(F) & 104 & 0.88 \\
UAU(Y) & 59 & 0.92 & AGU(S) & 19 & 0.67 & UUG(L) & 16 & 0.24 & UUC(F) & 132 & 1.12 \\
\hline
\end{tabular}

Highlights

The first complete mitochondrial genome sequence and annotation of Niviventer andersoni .

Phylogenetic relationships among major lineages of Muridae were reconstructed using mitochondrial genomes. 
figures/Figure1/Figure1-eps-converted-to.pdf 
figures/Figure2/Figure2-eps-converted-to.pdf 
figures/Figure3/Figure3-eps-converted-to.pdf 
figures/Figure4/Figure4-eps-converted-to.pdf 